\title{
Production of bioactive liver-targeting interferon Mu-IFN-CSP by soluble prokaryotic expression
}

\author{
Along Liul ${ }^{1,2,3 \dagger}$, Shuiqing Gui ${ }^{4 \dagger}$, Lun Zhang ${ }^{1,2 \dagger}$, Zhaoxia Chen ${ }^{1,2}$, Yanan Tang ${ }^{1,2}$, Mingzhu Xiao 1,2, Jie Wang ${ }^{1,2}$, \\ Wenbin Liu ${ }^{1,2}$, Xiaobao Jin ${ }^{1,2}$, Jiayong Zhu ${ }^{1,2}$ and Xuemei Lu ${ }^{1,2^{*}}$ (B)
}

\begin{abstract}
A novel liver-targeting interferon (IFN-CSP) was successfully over-expressed in our previous work. The in vitro and in vivo investigation revealed that IFN-CSP has significant anti-hepatitis B virus (HBV) effect and liver-targeting capacity. However, due to the IFN-CSP tends to form inclusion bodies in recombinant Escherichia coli (E. coli), efficient production of the soluble liver-targeting interferon is a challenge. In view of biomedical application, novel strategies for efficiently expressing liver-targeting interferon and overcoming its poor solubility are necessary and important. In the present study, a modified mu-IFN-CSP was designed base on the amino acid mutant of the native IFN-CSP. Meanwhile, the coding sequence of mu-IFN-CSP was optimized for E. coli preferred codon and the induction conditions for expression were optimized by an orthogonal test. After amino acid mutant, codon optimization and induction conditions optimization, the solubility of Mu-IFN-CSP in E. coli was up to $98.4 \%$. The structural comparison and molecular dynamic simulation showed that the Mu-IFN-CSP formed three structure changes and were more stable than the native IFN-CSP. Tissue sections binding assays revealed that Mu-IFN-CSP was also able to specific binding to liver. In vitro anti-HBV activity assays showed that the soluble Mu-IFN-CSP has improved anti-HBV effect in HepG2.2.15 cells compared to the native IFN-CSP. The present study reports for the first time that liver-targeting interferon MuIFN-CSP can be expressed as soluble form, and also contributes to further support its application as liver-targeting anti-HBV medicine.
\end{abstract}

Keywords: Liver-targeting interferon, Amino acid mutant, Preferred codon optimized, Escherichia coli, Soluble expression, Expression conditions optimized

\section{Introduction}

Interferon (IFN) is a kind of cytokines with the ability to induce antiviral, immunomodulatory and antitumor effects (Degasperi et al. 2017; Kotredes et al. 2017; Vu et al. 2016). IFNs have been applied as therapeutic medicine since first cloning and expressing interferon using DNA recombinant technology (Goeddel et al. 1980). However, some shortcomings such as

\footnotetext{
*Correspondence: Iuxuemei605@163.com

${ }^{\dagger}$ Along Liu, Shuiqing Gui and Lun Zhang contributed equally to this work as first authors

${ }^{2}$ Guangdong Provincial Key Laboratory of Pharmaceutical Bioactive Substances, 280 Wai Huan Dong Road, Guangzhou Higher Education Mega Center, Guangzhou 510006, People's Republic of China

Full list of author information is available at the end of the article
}

no organ-specific affinity have limited the therapeutic efficacy of interferon (Suginoshita et al. 2001). Molecular targeted drugs may provide promising strategies for new interferon design (Kato 2015). In our previous study, human IFN $\alpha 2 b$ have been successfully fused with Plasmodium region I peptide to construct a novel liver-targeting fusion interferon (IFN-CSP) (Lu et al. 2014). Use HepG2.2.15 cells and HBV-transgenic mice as in vitro and in vivo model, the investigation revealed that IFN-CSP has significant anti-hepatitis B virus (HBV) effect ( $\mathrm{Lu}$ et al. 2015a, b). The in vitro and in vivo study verified that the novel liver-targeting interferon specific targeting to liver tissue ( $\mathrm{Lu}$ et al. 
2016; Wang et al. 2015). Thus, the novel liver-targeting interferon may be an excellent substitute for IFN $\alpha 2 b$ as anti-HBV medicine.

As the interferon genes do not have introns (Radhakrishnan et al. 1996) and Escherichia coli (E. coli) can grow rapidly, the majority of human interferon has been expressed using recombinant $E$. coli as host cell up to now. IFN $\alpha 2 b$ contains 165 amino acids, and cysteines at position 1 and 98 as well as 29 and 138 formed two disulfide bonds (Gull et al. 2013). Overexpression of recombinant IFN $\alpha 2 b$ often result in protein misfolding in E. coli cytoplasm, and consequent aggregation into inclusion bodies, which are insoluble and usually biologically inactive (Neves et al. 2004). The downstream protein refolding procedures from inclusion bodies are quite cumbersome and low efficiency (Valente et al. 2004). Since IFN-CSP is a fusion protein combining plasmodium region I peptide with human IFN $\alpha 2 \mathrm{~b}$, the problem in the soluble expression of the novel liver-targeting interferon in $E$. coli and the recombinant IFN $\alpha 2 \mathrm{~b}$ remains the same. For biomedical application in livertargeting anti-HBV medicine, efficiently expressing livertargeting interferon and overcoming its poor solubility are very important.

In the present study, a modified mu-IFN-CSP was designed and synthesized based on the amino acid position mutant of the native IFN-CSP. Meanwhile, the coding sequence of mu-IFN-CSP was optimized for $E$. coli preferred codon and the induction conditions for expression were optimized by an orthogonal test. To clarify the structure-function relationships and investigate the influence of the mutation on the stability of the novel liver-targeting interferon, the threedimensional structural models of the native IFN-CSP and the mutant $\mathrm{Mu}$-IFN-CSP were constructed and the molecular dynamic simulations were conducted. We also compared the liver tissue binding capacity and in vitro anti-HBV effects of native IFN-CSP and mutant $\mathrm{Mu}-\mathrm{IFN}-\mathrm{CSP}$.

\section{Materials and methods}

\section{Plasmids and strains}

pMD20-T (Takara, Otsu, Japan) was employed to clone gene and pET-21b (Novagen, Madison, USA) was employed to construct expression plasmid. E. coli strain DH5 $\alpha$ (Novagen, Madison, USA) was employed for gene manipulation and BL21 (DE3; Novagen, Madison, USA) was employed to construct recombinant expression strain. Recombinant IFN-CSP/pET-21b expression plasmid was obtained from Guangdong Provincial Key Laboratory of Pharmaceutical Bioactive Substances, Guangzhou, People's Republic of China.

\section{Design, structural models construction and molecular dynamic simulation}

To improve the expression level and the poor solubility of IFN-CSP in recombinant E. coli, a new mutant was designed based on the native IFN-CSP using amino acid position mutant. Meanwhile, the coding sequence of muIFN-CSP was designed based on the preferred codon usage of E. coli (http://www.kazusa.or.jp/codon/). To provide convenient restriction enzyme sites for recombinant plasmid construct, NdeI/XhoI sites were inserted into the $5^{\prime}$ - and $3^{\prime}$-ends of the mu-IFN-CSP gene.

To compare the similarity of protein compositions between the native IFN-CSP and the mu-IFN-CSP, the amino acid composition assay was carried out. To clarify the structure-function relationships and study the influence of the mutation on IFN-CSP stability, the threedimensional structural models of the native IFN-CSP and the mutant Mu-IFN-CSP were constructed and the molecular dynamic simulations were conducted according to previous study (Bao et al. 2015). The known crystal structure of human IFN $\alpha 2 \mathrm{~b}$ (PDB ID: 1RH2), which is closely homologous to IFN-CSP, was used to establish the homologous model. The Swiss-PDB viewer software was used to model the three-dimensional structures of the native IFN-CSP and the mu-IFN-CSP. In order to obtain the optimal conformations, the two protein structure models were chosen as the initial coordinates for molecular dynamic (MD) simulations with AMBER 9.0 software package. During the simulation, the root mean square deviation (RMSD) values of backbone atoms relative to the minimized starting structure were used to evaluate the stability of the simulation. The binding free energies of the two protein systems were also monitored over the course of simulations.

\section{Construction of mu-IFN-CSP and recombinant plasmids}

The improved splicing by overlapping extension polymerase chain reaction (SOE-PCR) was applied to synthetize the mutant gene as described before (Lu et al. 2010, 2015b). 16 oligonucleotides were designed and the nucleotide sequences are presented in Table 1. After SOE-PCR, the synthesized full gene fragments of mu-IFN-CSP were recovered and linked to the pMD20-T vector as described before (Lu et al. 2010, 2015b). The constructed cloning plasmid mu-IFN-CSP/pMD20-T was digested by restriction enzyme and the target-gene fragments were ligated into the cloning site of the pET-21b vector according to the procedures described by the manufacturer (Fig. 4a).

\section{Protein expression and solubility optimization}

The generated mu-IFN-CSP/pET-21b plasmids were transformed into E. coli BL21 (DE3) to test the expression 
Table 1 Nucleotide sequences of oligonucleotides designed for assembly of mu-IFN-CSP

\begin{tabular}{ll}
\hline Primers & Nucleotide sequence (from $\mathbf{5}^{\prime}$ end to $\mathbf{3}^{\prime}$ end) \\
\hline MulC-1 & GGAATTCCATATGTGTGATCTGCCTCAGA \\
MulC-2 & GTGCCAGGAGAATCAAGGCACGACGGTACCCAGGCTGTGAGTCTGAGGCAGATCACA \\
MulC-3 & GCCTTGATTCTCCTGGCACAAATGCGTCGTATCTCTCCTTTCTCCTGCCTGAAGGACC \\
MulC-4 & TATCATCAAACTCCTCCTGTGGAATTCAAAGTATGACGGTCCTTCAGGCAGGAGAA \\
MulC-5 & CAGGAGGAGTTTGATGATAAACAGTTCCAGAAGGCTCAAGCCATCTCTGTCCTCCATG \\
MulC-6 & CTTTTGTGGTAAAGAGGTTGAAGATCTGCTGGATCATCTCATGGAGGACAGAGATGGC \\
MulC-7 & AACCTCTTTACCACAAAAGATTCATCTGCTGCTTGGGATGAGGACCTCCTTGACAAAT \\
MulC-8 & CTTCCAAGTCATTCAGCTGCTGGTAGAGTTCGGTGCAGAATTTGTCAAGGAGGTCCTC \\
MulC-9 & CAGCTGAATGACTTGGAAGCCTGTGTATGCAGGAGGAGCGTGTGGGAGAAACTCCAC \\
MulC-10 & AGTATTTCTTCACAGCCAAGATGGAGTCCGCATTCATCAGTGGAGTTTCTCCCACACG \\
MulC-11 & TTGGCTGTGAAGAAATACTCCGTCGTATCACTCTCTATCTGACAGAGAAGAAATACA \\
MulC-12 & GCATGATTTCTGCACGGACAACCTCCCAGGCACAAGGGCTGTATTTCTTCTCTGTCAG \\
MulC-13 & GTCCGTGCAGAAATCATGCGTTCCCTCTCTTATCAACAAACTTGCAAGAACGTTTAC \\
MulC-14 & CGTAATTCTCGTTGTCTCCTTACGACGTAAACGTTCTTGCAAGTT \\
MulC-15 & GACAACGAGAAATTACGTAAACCAAACATAAAAATTAAAGCAACCAGCGGA \\
MulC-16 & CCGCTCGAGACCATCCGCTGGTTGCTTTAA \\
\hline
\end{tabular}

Letters in bold type denote the overlapped parts in Mu-IFN-CSP. Restriction sites are underlined

and solubility. The culture medium for the recombinant strains is Luria-Bertani (LB) and the conditions and the process for protein expression are as described before ( $\mathrm{Lu}$ et al. 2010, 2015b). The induced recombinant strains were disrupted by ultrasonication for $10 \mathrm{~min}$ in $50 \mathrm{mM}$ Tris$\mathrm{HCl}$ buffer (30\% sucrose, $2.5 \mathrm{mM}$ DTT, $1 \mathrm{mM}$ EDTA, $\mathrm{pH}$ 8.0). The strain cell lysate was centrifuged (8000g, $4{ }^{\circ} \mathrm{C}, 30 \mathrm{~min}$ ) to separate the precipitate and soluble parts. To analyze the expression levels and solubility of recombinant protein, $15 \%$ sodium dodecyl sulfate polyacrylamide gel electrophoresis (SDS-PAGE) was used. To characterize the antigenicity of the mu-IFN-CSP, western blot analysis was performed, in which the primary antibody and the second antibody were used as previously described ( $\mathrm{Lu}$ et al. 2015b).

To improve the expression level and the solubility of mutant in $E$. coli, the induction conditions like induction timing, induction temperature, IPTG concentrations, induction time were optimized by an orthogonal experiment $(\mathrm{L}(25)(5)(4))$. Different conditions of induction include $\mathrm{OD}_{600}(0.3,0.6,0.9,1.2,1.5)$, temperatures $\left(25,28,31,34,37^{\circ} \mathrm{C}\right)$, IPTG concentrations (0.1, 0.2, 0.4, 0.6, $0.8 \mathrm{mM})$ and induction times $(2,4,6,8,10 \mathrm{~h})$. Samples $(15 \mu \mathrm{L} /$ lane) were examined by $15 \%$ SDS-PAGE and Gel-Pro analyzer Version 4.5 software was used to quantify the percentage fraction of mu-IFN-CSP by densitometry. The solubility level was calculated as: solubility $=\mathrm{S}^{\prime} /$ $\left(\mathrm{S}^{\prime}+\mathrm{P}^{\prime}\right)$, where $\mathrm{S}^{\prime}$ is the amount of mu-IFN-CSP in the soluble supernatant $(\mathrm{S})$, and $\mathrm{P}^{\prime}$ is the amount of mu-IFNCSP in the insoluble precipitation $(\mathrm{P})$.

\section{Protein purification}

Due to its His Tag, the mu-IFN-CSP was purified by affinity chromatography with Hispatch-chelating agarose (GE healthcare, Wisconsin, USA). The soluble supernatant was loaded into the HisTrap affinity column, and the conditions and the process for protein purification are as described before (Lu et al. 2010, 2014). To remove endotoxin, the protein sample was applied to polymyxin B column (Bio-Rad, CA, USA), and the lipopolysaccharides (LPS) content of the sample was detected using chromogenic limulus amoebocyte lysate assay (Associates of Cape Cod, MA, USA). The purity of the purified mu-IFN-CSP was assessed by reverse phase high-performance liquid chromatography (RP-HPLC) as described before (Lu et al. 2014, 2015b).

\section{Tissue sections preparation and binding assays}

For liver tissue binding capacity comparison of native IFN-CSP and mutant Mu-IFN-CSP, the immunofluorescence analyses were performed. Balb/c mice (20-25 g) were purchased from the Center for Experimental Animals of Guangdong Province (Guangzhou, China). All research procedures involving mice were approved by the Guidelines for the Care and Use of Experimental Animals, the Guangdong Pharmaceutical University (SYXK (Yue) 2012-0125) and the Guangdong Pharmaceutical University Animal Care and Use Committee, China. The tissue sections preparation, immunofluorescence experiment and binding assays were performed as described before (Lu et al. 2014). 


\section{In vitro antiviral experiments}

The in vitro antiviral activities of native IFN-CSP and muIFN-CSP were evaluated using HepG2.2.15 cell as model. The cell culture conditions and the process of in vitro antiviral experiment are as described before $(\mathrm{Lu}$ et al. 2014, 2015a). The treating time is 6 days and the concentration of native IFN-CSP and mu-IFN-CSP was the same (at final concentrations of $0.3 \mathrm{nmol} / \mathrm{L}$ ). The cells were collected for intracellular HBV-DNA and hepatitis B s antigen (HBsAg) expression assays. The culture supernatants were collected for HBV-DNA and HBV antigens assays.

\section{Measurement of HBsAg, $\mathrm{HBeAg}$ and HBV-DNA}

The enzyme-linked immunosorbent assay (ELISA) was used to measure HBsAg and hepatitis $\mathrm{B}$ e antigen (HBeAg) according to the manufacturer's instructions of HBsAg and HBeAg diagnostic kits (Shanghai Kehua Biotech Co. Ltd., Shanghai, China). The inhibitory level was calculated as: Inhibitory rate $(\%)=\left(1-\mathrm{OD}_{\mathrm{IFN}}\right.$ CSP or Mu-IFN-CSP $\left./ \mathrm{OD}_{\text {control }}\right) \times 100 \%$. The real-time fluorescent quantification PCR (FQ-PCR) was used to measure the copies of HBV-DNA according to the manufacturer's recommendations of HBV-DNA test kit (Da-An Gene Co., Guangzhou, China). The inhibitory level was calculated as: Inhibitory rate $(\%)=\left(1-\right.$ HBV-DNA copies IFN-CSP or Mu-IFN-CSP $_{\text {HBV- }}$ DNA copies control) $_{1} \times 100 \%$.

\section{Immunofluorescent analysis for $\mathrm{HBsAg}$}

HBsAg expression was also analyzed by immunofluorescence analysis as described before (Lu et al. 2015a). The treated HepG2.2.15 cells were seeded on coverslip and the primary antibody and the second antibody were goat polyclonal anti-HBsAg antibody (1:200; Novus Biologicals, CO, USA) and Alexa Fluor Cy3-conjugated donkey anti-goat IgG (1:200; Beyotime, Guangzhou, China), respectively. 4',6-diamidino-2-phenylindole (DAPI) was used for the cells nuclear staining. Finally, fluorescence microscope (Leica, Heidelberg, Germany) was used for immunofluorescence analyses and images capture.

\section{Statistical analysis}

The values of HBsAg, HBeAg and HBV-DNA were expressed as the mean \pm standard error of the mean (SEM). The SPSS (version 13.0 for Windows) statistical software was used for statistical analyses. One-Way Analysis of Variance (ANOVA) was used to analyze the differences between mean values. $P<0.05$ is considered statistically significant.

\section{Results}

Design, structural models construction and molecular dynamic simulation

To obtain a modified liver-targeting interferon with enhanced solubility compared to the native IFN-CSP, we designed a new mutant Mu-IFN-CSP (GenBank Accession No. ALP75560.1) using amino acid position mutant. The mutant contains 185 amino acids, among which 27 amino acids are mutations compared to the native IFN-CSP (Fig. 1). Meanwhile, to eliminate the adverse influence of rare codons on recombinant expression of $\mathrm{Mu}$-IFN-CSP in E. coli, the DNA sequence of the mutant gene (GenBank Accession No. KP719982.1) was analyzed and codon-optimized according to the preferred codon usage of E. coli (Fig. 1).

To analyze the key positions of mu-IFN-CSP, the structural models of native IFN-CSP and mu-IFN-CSP were constructed and compared. The comparison result of the amino acid sequences of native protein and mutant was showed in Fig. 2A, where 27 mutation positions were highlighted. Structure alignment of native IFN-CSP and Mu-IFN-CSP was showed in Fig. 2B. Compared with the native protein, the mutant formed three structure changes, there are two random coils turned into alpha helixes (Fig. 2B a, c) and one alpha helixes turned into random coil (Fig. 2B b). The results of RMSDs showed that both the native IFN-CSP and mu-IFN-CSP simulation systems reached equilibrium after $2 \mathrm{~ns}$ and the RMSD fluctuations were very small in the remaining $2 \mathrm{~ns}$ process (Fig. 2C). The total energy of native IFN-CSP and $\mathrm{Mu}$-IFN-CSP during MD simulations was showed in Fig. 2D. The energy curves of both the native IFN-CSP and mu-IFN-CSP were smooth. The total energy values of the native IFN-CSP and mu-IFN-CSP systems were $-8.8 \times 10^{4}$ and $-9.0 \times 10^{4} \mathrm{kcal} / \mathrm{mol}$, respectively.

\section{Construction of mu-IFN-CSP and recombinant plasmids}

The mu-IFN-CSP gene was constructed by improved SOE-PCR method. The Fig. 3a showed the first and the second PCR products ranging $66-97$ and $135-175 \mathrm{bp}$ in size, respectively. The full-length gene of fusion mu-IFNCSP is $577 \mathrm{bp}$ in size (Fig. 3a Lane 13). The recombinant plasmid mu-IFN-CSP/pMD20-T was identified by PCR screening, DNA sequencing and restriction endonuclease analysis (Fig. 3b). After digested with $\mathrm{NdeI} / \mathrm{XhoI}$, the gene fragment of mu-IFN-CSP was ligated into the cleaved plasmid to construct recombinant mu-IFN-CSP/ pET-21b, and recombinant plasmid was verified by the sequencing data. 
1 TGT GAT CTG CCT CAG ACT CAC AGC CTG GGT AAC CGT CGT GCC TTG

$\begin{array}{lllllllllllllll}\text { C } & \mathbf{D} & \mathrm{L} & \mathrm{P} & \mathrm{Q} & \mathrm{T} & \mathrm{H} & \mathrm{S} & \mathrm{L} & \mathrm{G} & \mathbf{N} & \mathrm{R} & \mathrm{R} & \mathrm{A} & \mathrm{L}\end{array}$

46

ATT CTC CTG GCA CAA ATG CGT CGT ATC TCT CCT TTC TCC TGC CTG

$\begin{array}{lllllllllllllll}I & L & L & A & Q & M & R & R & I & S & F & F & S & C & L\end{array}$

91 AAG GAC CGT CAT GAC TTT GAA TTC CCA CAG GAG GAG TTT GAT GAT $\begin{array}{lllllllllllllll}\mathrm{K} & \mathrm{D} & \mathrm{R} & \mathrm{H} & \mathrm{D} & \mathrm{F} & \mathrm{E} & \mathrm{F} & \mathrm{P} & \mathrm{Q} & \mathrm{E} & \mathrm{E} & \mathrm{F} & \mathbf{D} & \mathrm{D}\end{array}$

136 AAA CAG TTC CAG AAG GCT CAA GCC ATC TCT GTC CTC CAT GAG ATG $\begin{array}{lllllllllllllll}\mathbf{K} & \mathbf{Q} & \mathbf{F} & \mathbf{Q} & \mathrm{K} & \mathrm{A} & \mathbf{Q} & \mathbf{A} & \mathrm{I} & \mathrm{S} & \mathrm{V} & \mathrm{L} & \mathbf{H} & \mathbf{E} & \mathbf{M}\end{array}$

181 ATC CAG CAG ATC TTCAAC CTC TTT ACC ACAAAA GAT TCA TCT GCT

$\begin{array}{lllllllllllllll}\text { I } & Q & Q & I & F & \mathbf{N} & \mathrm{L} & \mathbf{F} & \mathrm{T} & \mathrm{T} & \mathrm{K} & \mathbf{D} & \mathrm{S} & \mathrm{S} & \mathrm{A}\end{array}$

226 GCT TGG GAT GAG GAC CTC CTT GAC AAA TTC TGCACC GAA CTC TAC
A
E D
L $\mathrm{L}$
D $\mathbf{K}$
C
$\begin{array}{llll}E & L & Y\end{array}$

271 CAG CAG CTGAAT GAC TTG GAA GCC TGT GTG ATG CAG GAG GAG CGT
Q $\quad$ Q $\quad$ L $\quad$ N

316 GTG GGA GAAACT CCA CTG ATGAAT GCG GAC TCC ATC TTG GCT GTG
V G
E T P
L $\quad$
$\mathrm{N}$

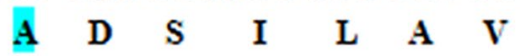

361 AAG AAA TAC TTC CGT CGT ATC ACT CTC TAT CT GCA GAG AAG AAA
$\begin{array}{lllllllllllllll}\mathbf{K} & \mathbf{K} & \mathbf{Y} & \mathbf{F} & \mathbf{R} & \mathbf{R} & \mathbf{I} & \mathbf{T} & \mathbf{L} & \mathbf{Y} & \mathbf{L} & \mathbf{T} & \mathbf{E} & \mathbf{K} & \mathbf{K}\end{array}$

406 TAC AGC CCT TGT GCC TGG GAG GTT GTC CGT GCA GAA ATC ATG CGT

$$
\begin{array}{lllllllllllllll}
\mathbf{Y} & \mathbf{S} & \mathbf{P} & \mathbf{C} & \mathbf{A} & \mathbf{W} & \mathbf{E} & \mathbf{V} & \mathbf{V} & \mathbf{R} & \mathbf{A} & \mathbf{E} & \mathbf{I} & \mathbf{M} & \mathbf{R}
\end{array}
$$

451 TCC CTC TCT TTATCAACA AAC TTG CAA GAA CGT TTA CGT CGT AAG
$\begin{array}{lllllllllllllll}\mathbf{S} & \mathbf{L} & \mathbf{S} & \mathbf{L} & \mathbf{S} & \mathbf{T} & \mathbf{N} & \mathbf{L} & \mathbf{Q} & \mathbf{E} & \mathbf{R} & \mathbf{L} & \mathbf{R} & \mathbf{R} & \mathbf{K}\end{array}$

496 GAA GAC AAC GAG AAA TTA CGT AAA CCAAAA CAT AAA AAA TTAAAG

$\begin{array}{lllllllllllllll}\text { E } & D & \text { N } & \text { E } & \text { K } & \text { L } & \text { R } & \text { K } & \text { P } & \text { K } & \text { H } & \text { K } & \text { K } & \text { L } & \text { K }\end{array}$

541 CAA CCA GCG GAT GGT

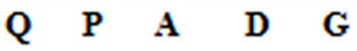

Fig. 1 The amino acid sequence and gene sequence of Mu-IFN-CSP. The amino acid residues of Mu-IFN-CSP in the blue shadow are mutation positions. The codons of Mu-IFN-CSP gene in frame are optimized

\section{Protein expression and solubility optimization}

The plasmid mu-IFN-CSP/pET-21b was transformed into E. coli BL21 (DE3) for protein expression. After induced with IPTG $(1.0 \mathrm{mM} / \mathrm{L}$ culture) for $4 \mathrm{~h}$, the recombinant protein with $21.5 \mathrm{kD}$ in size was successfully expressed with a $75.2 \%$ solubility (Fig. 4b). The result of western blot analysis also shows a specific band in the total protein from recombinant $E$. coli after induction compared with before induction (Fig. 4c).

Different induction conditions may have great impacts on the solubility and the expression level of recombinant protein in E. coli. In present experiment, four parameters including induction timing, induction temperature, IPTG concentrations and induction time were investigated by an orthogonal test. The results showed that the most optimal condition was at $\mathrm{OD}_{600}=0.9$, inducing the culture at $34{ }^{\circ} \mathrm{C}$ for $6 \mathrm{~h}$ with $0.1 \mathrm{mM}$ IPTG (Fig. 5). The solubility of Mu-IFN-CSP in E. coli was up to $98.4 \%$ (Figs. 5b, 6c).

\section{Protein purification}

To obtain mu-IFN-CSP, the soluble supernatant was subjected to a four-step purification process. The first step is affinity chromatography using hispatch-chelating agarose. Figure $6 \mathrm{c}$ Lane 4 shows the eluted protein fractions using $100 \mathrm{mM}$ imidazole and Fig. 6c Lane 5 shows 


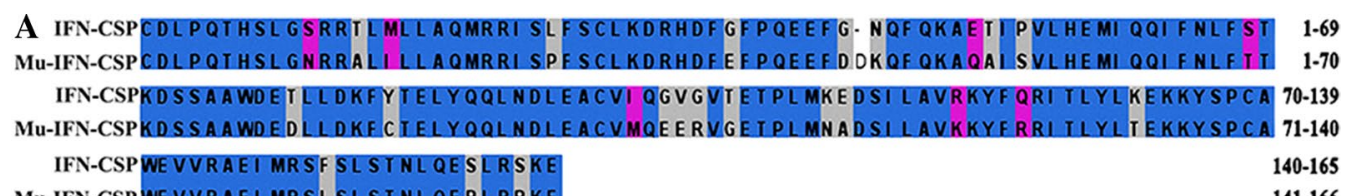
MU-IFN-CSP WE VVRAEI MRSL SL SINL QERLRRKE

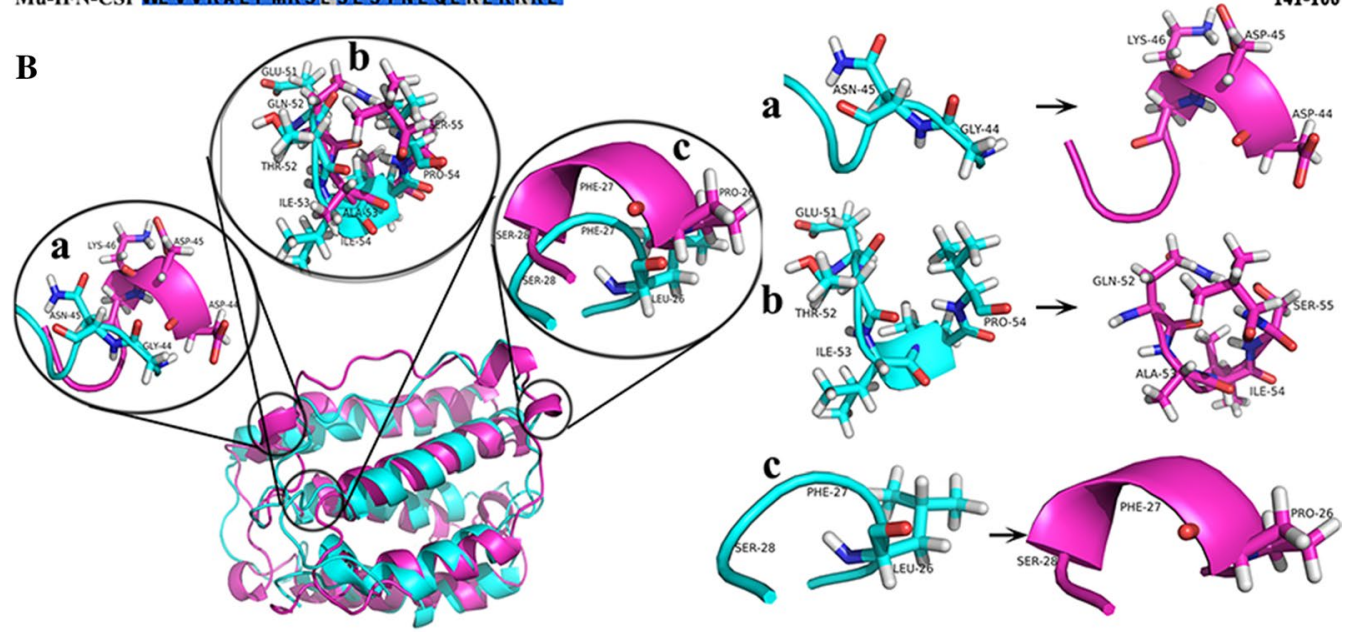

C

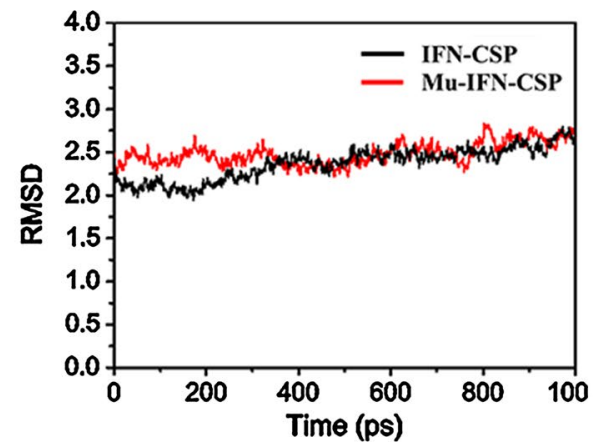

D

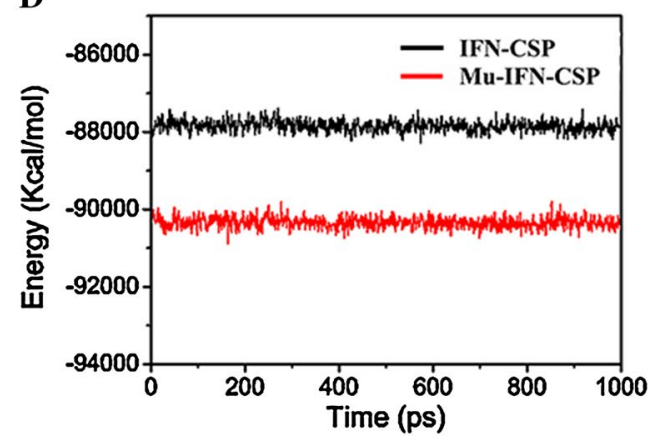

Fig. 2 Sequences and structure alignment of native IFN-CSP and Mu-IFN-CSP. A Amino acid sequences alignment of native IFN-CSP and MuIFN-CSP. The amino acid residues in the blue color regions are identical. The amino acid residues in other color regions are mutation positions. B Structure alignment of native IFN-CSP and Mu-IFN-CSP. Green denotes native IFN-CSP; red denotes Mu-IFN-CSP. Compared with native IFN-CSP, the Mu-IFN-CSP formed three structure changes (a, b and c). C The RMSDs of the molecular dynamic simulation systems. D The total energy of native IFN-CSP and Mu-IFN-CSP during MD simulations

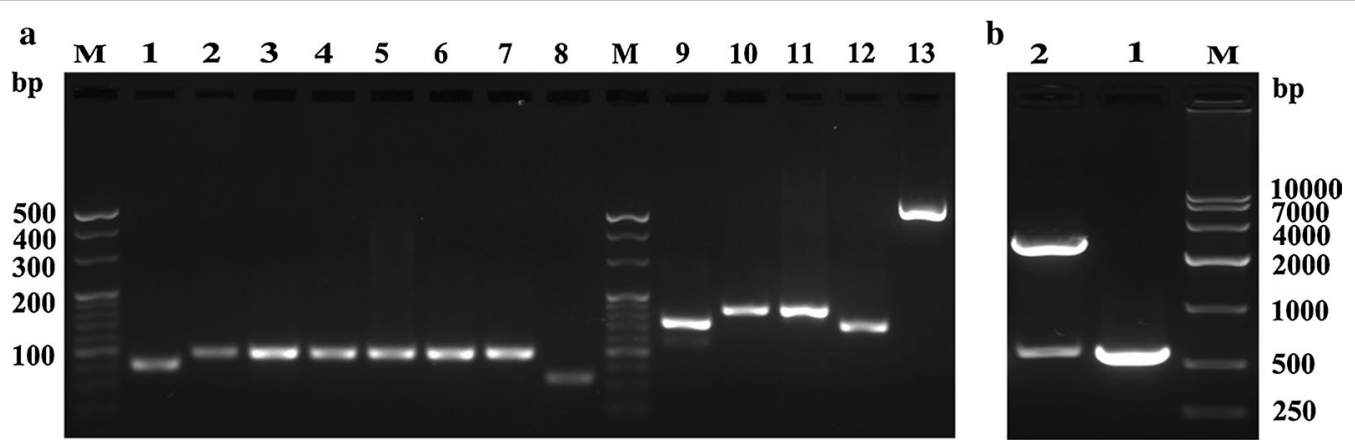

Fig. 3 Construction and identification of fusion gene mu-IFN-CSP. a Construction of fusion gene mu-IFN-CSP. Lanes 1-8 the first PCR products ranging 66-97 bp in size. Lanes 9-12 the second PCR products ranging 135-175 bp in size. Lane 13 the full-length fusion gene mu-IFN-CSP. Lane M DNA molecular weight marker. b Identification of fusion gene mu-IFN-CSP. Lane 1 PCR products of recombinant plasmids. Lane 2 recombinant plasmids mu-IFN-CSP/pMD20-T digested with Ndel/Xhol. Lane M DNA molecular weight marker 


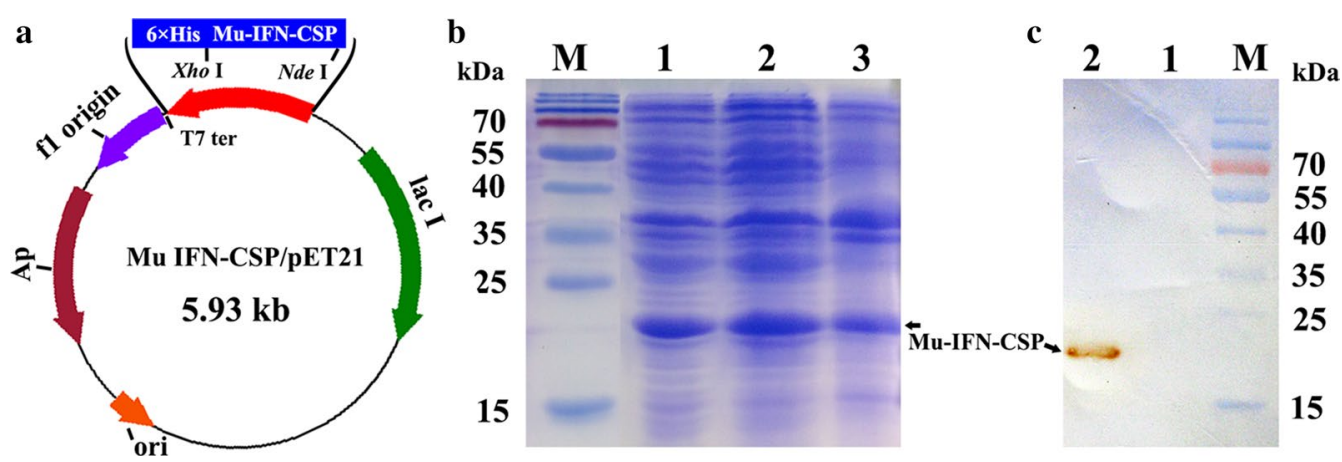

Fig. 4 Schematic diagram of mu-IFN-CSP gene in the expression vector mu-IFN-CSP/pET-21b and expression of mu-IFN-CSP protein in recombinant E. coli. a A schematic diagram of mu-IFN-CSP/pET-21b. b SDS-PAGE analysis of mu-IFN-CSP expression. Lane 1 total proteins of recombinant $E$. coli BL21 after IPTG induction. Lanes 2-3 supernatant and precipitation after ultrasonication and centrifugation. Lane M protein molecular weight marker. c Recombinant mu-IFN-CSP was analyzed by western blot analysis. Lanes 1-2 total proteins of E. coli BL21/pET-21b-mu-IFN-CSP before and after induction. Lane M protein molecular weight marker

\section{$\mathbf{a}$}
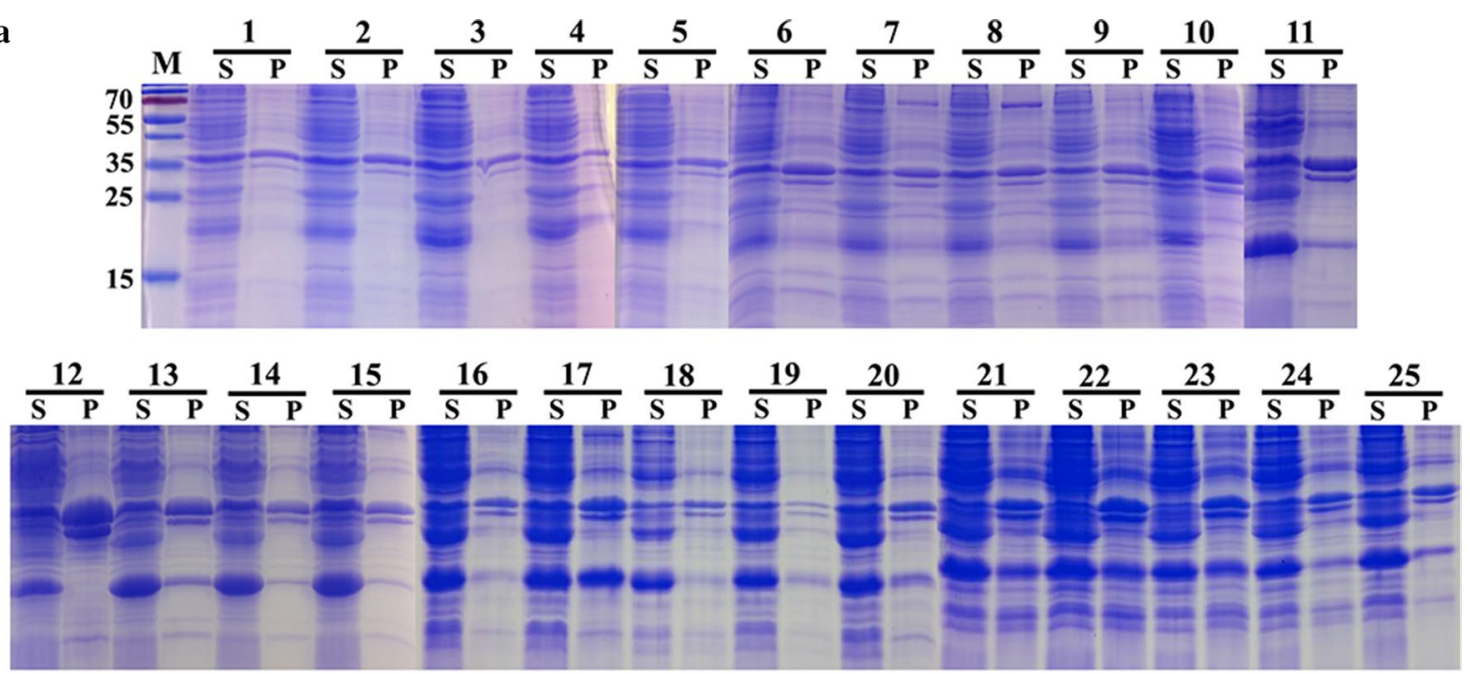

b

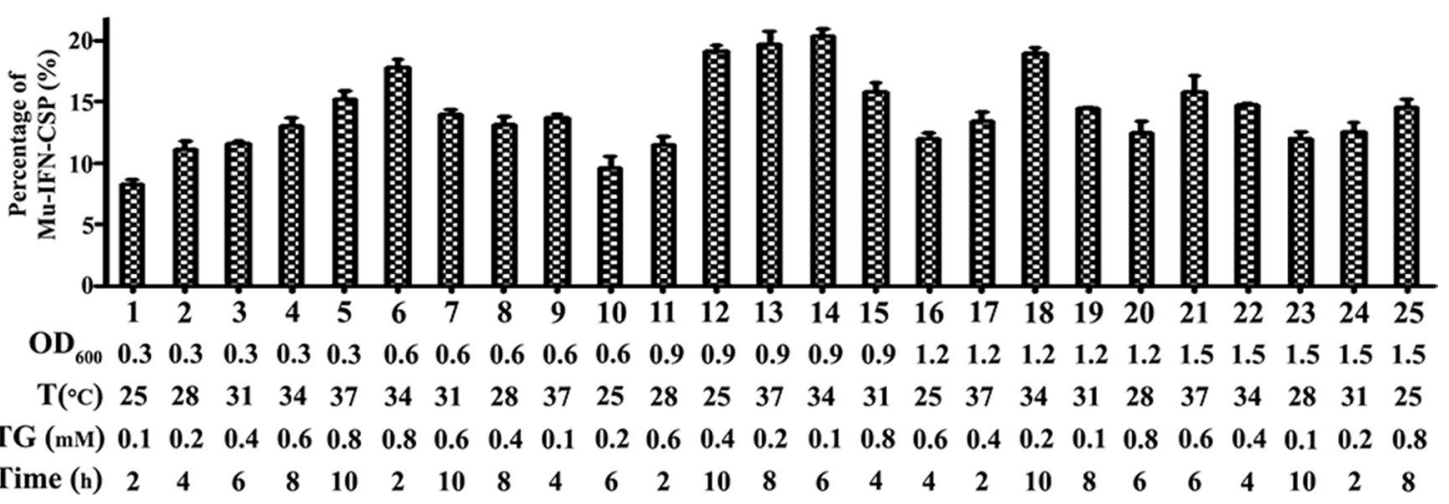

Fig. 5 Optimization of mu-IFN-CSP expression by orthogonal test. a SDS-PAGE analyses of mu-IFN-CSP expression at different induction conditions. Lane M protein molecular weight marker; $S$ soluble supernatant after cell disruption; $P$ insoluble precipitation. $\mathbf{b}$ Percentage of mu-IFN-CSP in the total soluble proteins was calculated by the target bands in SDS-PAGE (a) 

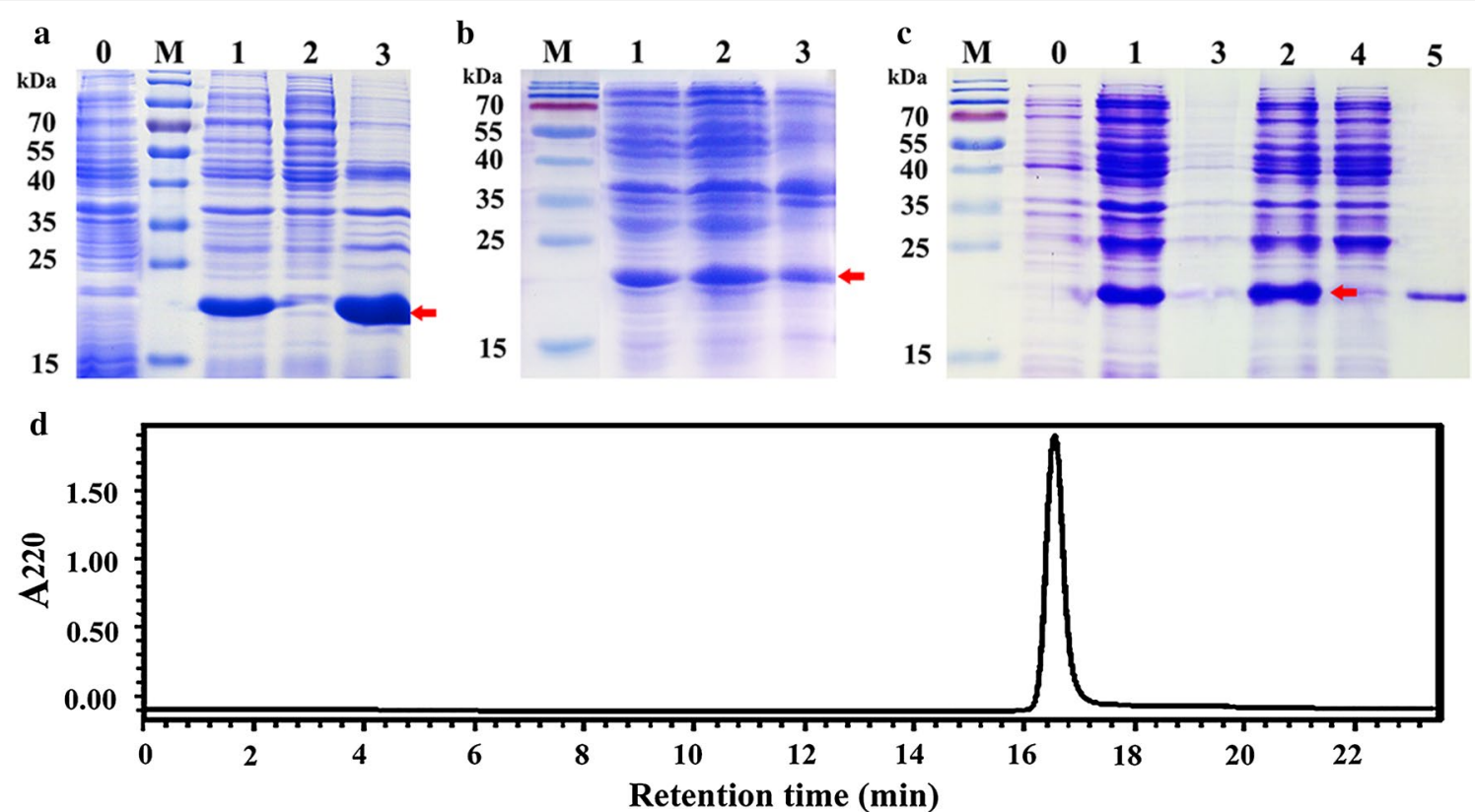

Fig. 6 Comparison of the solubility and characterization of recombinant protein. a Assay of expression and solubility of IFN-CSP in recombinant $E$. coli. b Assay of expression and solubility of mu-IFN-CSP in recombinant E. coli before optimization. c Assay of expression and solubility of mu-IFNCSP in recombinant E. coli after optimization and purification of mu-IFN-CSP. Lane M protein molecular weight marker. Lanes 0-1 total proteins of recombinant $E$. coli before and after induction. Lanes $2-3$ supernatant and precipitation after ultrasonication and centrifugation. Lane 4 eluted protein fraction using HisTrap affinity chromatography. Lane 5 purified mu-IFN-CSP using HisTrap affinity chromatography. The arrows indicate the intense bands at $21.5 \mathrm{kDa}$ corresponding to the target protein. $\mathbf{d}$ Analysis of purified IFN-CSP by RP-HPLC with a C18 column

the purified mu-IFN-CSP using $500 \mathrm{mM}$ imidazole. The following steps are desalting and endotoxin removing. The eluted product was purified by desalted dialysis and polymyxin $B$ column purification to avoid the negative influence of imidazole and LPS on the bioactivity of mu-IFN-CSP. The forth step is freeze-drying. After polymyxin B column purification, the LPS content in the purified product was less than $0.5 \mathrm{Eu} / \mathrm{mg}$ proteins. Figure $6 \mathrm{~d}$ showed that the RP-HPLC result of mu-IFN-CSP indicating the purity of the protein was over $98 \%$. The yield of the pure soluble mu-IFN-CSP is approximately $64.58 \mathrm{mg} / \mathrm{L}$ of $E$. coli culture.

\section{Tissue sections preparation and binding assays}

To study whether mutant was also able to specific binding to liver, both native protein and mutant were directly applied to liver slices and the immunofluorescence analyses were performed. Figure 7 shows photographs of fluorescent labeling of liver after native IFN-CSP or mu-IFN-CSP incubation. Compared with the control (Fig. 7A), both native IFN-CSP (Fig. 7B) and mu-IFN-CSP (Fig. 7C) treatment displayed green fluorescence, which along the basolateral region and sinusoidal borders.
Effect of native IFN-CSP and mu-IFN-CSP on HBV antigens secretion

The HBsAg and HBeAg in the culture supernatant were measured by ELISA. After treated with native IFN-CSP and mu-IFN-CSP, HBsAg (Fig. 8a) and HBeAg (Fig. 8b) secretion significantly reduced. The inhibition ratios of native IFN-CSP and mu-IFN-CSP on HBsAg were 52.01 and $63.80 \%$, respectively. And the inhibition ratios of native IFN-CSP and mu-IFN-CSP on HBeAg were 31.79 and $42.39 \%$, respectively.

\section{Effect of native IFN-CSP and mu-IFN-CSP on HBV-DNA}

HBV-DNA in culture supernatant and intracellular of HepG2.2.15 cells was measured to further verify the anti-HBV effect of native IFN-CSP and mu-IFN-CSP. The results revealed that culture supernatants (Fig. 8c) and intracellular HBV-DNA (Fig. 8d) of the treated group were significantly decreased. The inhibition ratios of native protein and mutant on HBV-DNA in supernatant were 56.77 and $69.09 \%$, respectively. For intracellular HBV-DNA, the inhibition ratios of native IFN-CSP and mu-IFN-CSP were 37.89 and 56.31\%, respectively. 

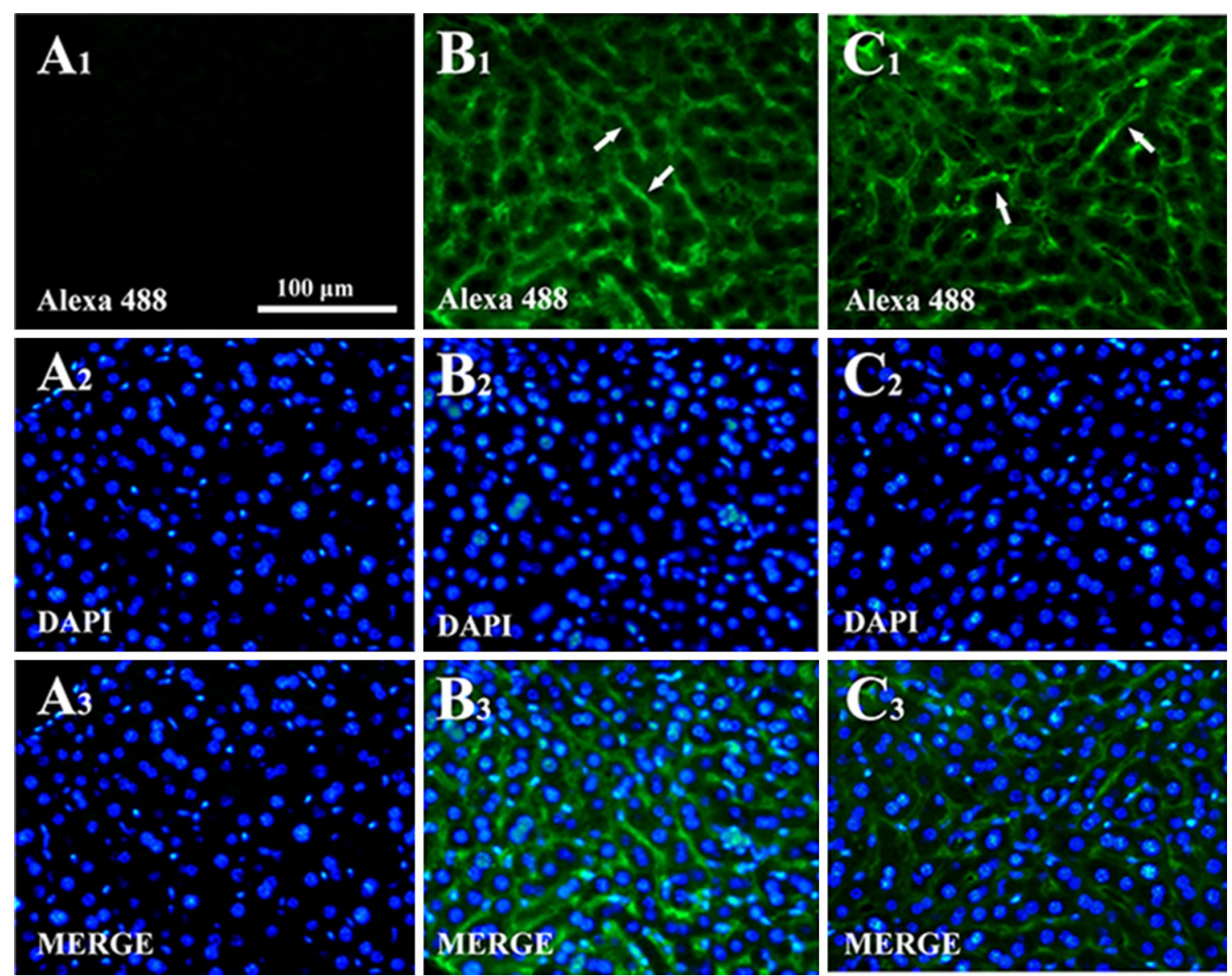

Fig. 7 Fluorescence photomicrographs of liver tissue after incubated with IFN-CSP or mu-IFN-CSP. A Controls; B IFN-CSP; C mu-IFN-CSP; 1 green fluorescent labeling of protein stained with anti-IFN antibody; $\mathbf{2}$ blue nuclear stained with DAPI; $\mathbf{3}$ merged images of 1 and 2 . Arrows indicate examples of distinct green fluorescent labeling of IFN-CSP and mu-IFN-CSP. Calibration bar $=100 \mu \mathrm{m}$ for all images

\section{Effect of native IFN-CSP and mu-IFN-CSP on HBsAg expression}

The immunofluorescence analysis was used to study the HBsAg expression in HepG2.2.15 cells. The fluorescence images captured by microscope showed that the signal of HBsAg is expressed in the cytoplasm and membrane of the HepG2.2.15 cells (Fig. 9). Compared with the control cells, native IFN-CSP and mu-IFN-CSP treated obviously suppress the expression of HBsAg.

\section{Discussion}

The novel liver-targeting interferon IFN-CSP is a promising anti-HBV protein (Lu et al. 2015a, b). However, its efficient production for biomedical applications has been hampered by insoluble inclusion bodies in recombinant $E$. coli expression system and the cumbersome protein refolding process. Intend to express soluble, active recombinant liver-targeting interferon in $E$. coli, several strategies have been used in the expression of natively folded liver-targeting interferon in the present study, including amino acid positions mutation, E. coli preferred codon optimize and induction conditions optimize.

At first, based on the native IFN-CSP, we have designed a modified mu-IFN-CSP using amino acid positions mutant. Figure $2 \mathrm{~A}$ shows the comparison result for the amino acid sequence of the native protein and the mutant, where 27 amino acid positions are mutations. The homology comparison of amino acid sequences of IFN region in the mu-IFN-CSP showed the $100 \%$ homology with reported amino acid sequences of human IFN $\alpha$ B/D hybrid (Ghaffar et al. 1992; Horisberger and de Staritzky 1987). The amino acids $1-60$ of human IFN $\alpha \mathrm{B} / \mathrm{D}$ hybrid come from IFN $\alpha \mathrm{B}$ and the amino acids $61-166$ of IFN $\alpha \mathrm{B} / \mathrm{D}$ come from IFN $\alpha \mathrm{D}$ (Meister et al. 1986). The IFN $\alpha$ B/D hybrid has been found to have comparable or higher antiviral efficacy against vesicular stomatitis virus (VSV), bovine viral diarrhea virus (BVDV) and herpes simplex virus type 1 (HSV-1/VR3) than the 

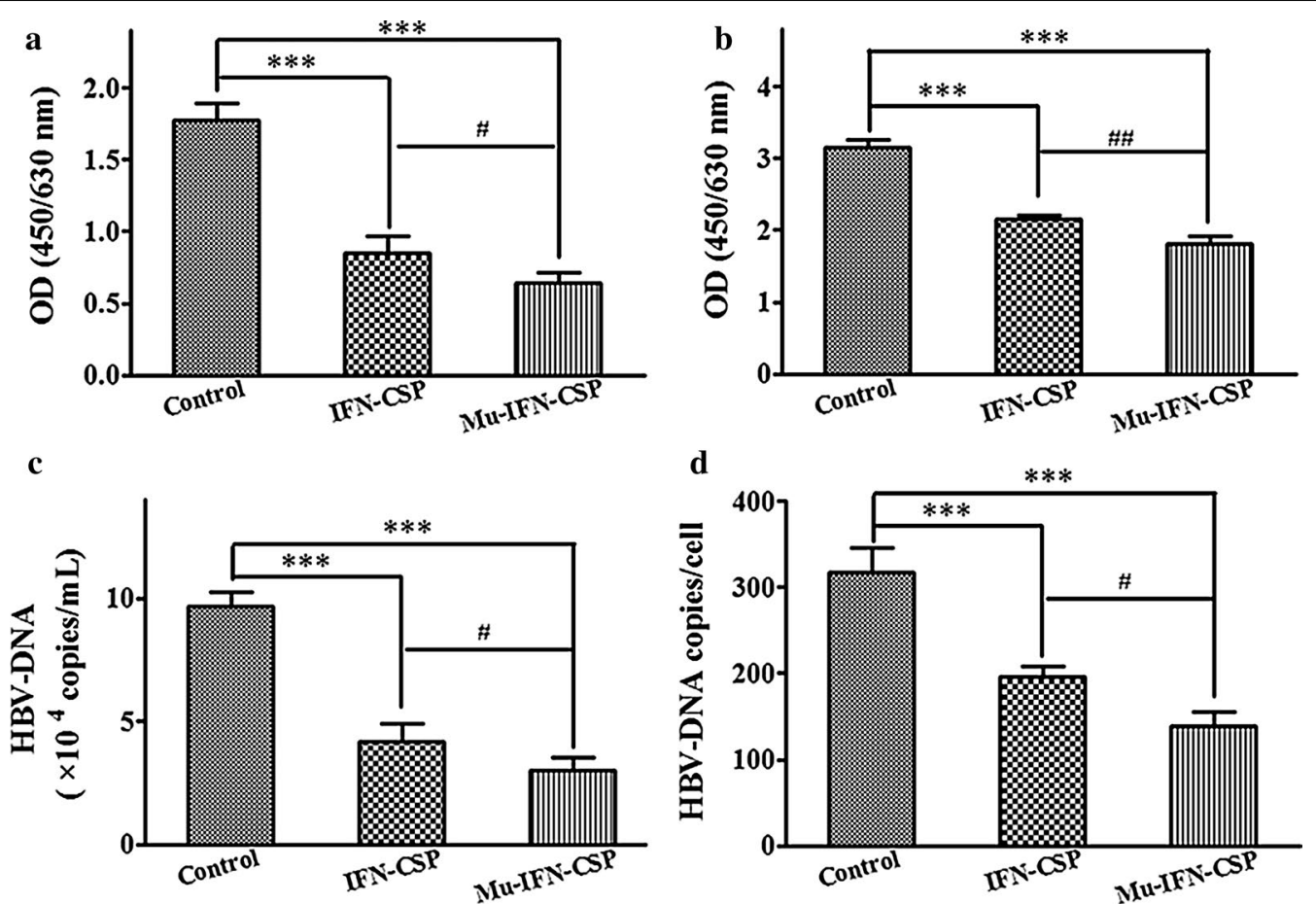

Fig. 8 Effect of IFN-CSP or mu-IFN-CSP on HBV antigens secretion and HBV-DNA replication in HepG2.2.15 cells. HepG2.2.15 cells were cultured in the presence of IFN-CSP or mu-IFN-CSP for 6 days. Hepatitis B surface antigen ( $\mathrm{HBsAg}$; a) and hepatitis B e antigen (HBeAg; b) in the culture supernatants were analyzed by enzyme-linked immunosorbant assay (ELISA). Supernatant HBV-DNA (c) and intracellular HBV-DNA (d) were measured by real-time quantitative PCR. Data represent the mean \pm SEM of three experiments. ${ }^{* * *} P<0.001$ drug group vs control group; ${ }^{\#} P<0.05$, ${ }^{\#} P<0.01$ IFN-CSP group vs mu-IFN-CSP group

parents IFN $\alpha 2 \mathrm{a}$, IFN $\alpha \mathrm{B}$ and IFN $\alpha \mathrm{D}$ (Gangemi et al. 1989; Peek et al. 2004). Structure-function investigations of IFN $\alpha$ have found that the differences in activity were correlated with their ability to bind the receptor, and several distinct sequence regions may involve the interactions between ligand and receptor or ligand and ligand (Karpusas et al. 1997; Meister et al. 1986; Radhakrishnan et al. 1996). Comparison of the mu-IFN-CSP with the native IFN-CSP shows that there are three structure differences including two random coils turned into alpha helixes and one alpha helix turned into random coil (Fig. 2B). Figure 2D shows the energy curves and total energy values, which indicate that the mu-IFN-CSP were more stable than the native IFN-CSP. Compared to the native IFN-CSP with $0-2.6 \%$ solubility (Fig. 6a) (Lu et al. 2014, 2015b), the solubility of mu-IFN-CSP in E. coli was $75.2 \%$ before induction conditions optimized (Fig. 6b). In vitro anti-HBV activity study in HepG2.2.15 cells showed that the soluble Mu-IFN-CSP has improved inhibition effect on HBV replication compared to the native IFN-CSP. In this, we speculate that these mutations in $\mathrm{Mu}$-IFN-CSP may relate to not only the activity but also the stability and the solubility.
Previous studies have found that the rare codons have the greatest degree of conformity to lowly expressed genes in the E. coli cell, and the expression level may significantly be improved by codon optimization (Sharp and Li 1987; Peng et al. 2004; Xu et al. 2006). There are 18 amino acids in the Mu-IFN-CSP encoded by the rare codons of E. coli. Among these, R12, R13, R23, R33, R105, R125, R126, R145, R150, R161, R163, and R164 were encoded by AGG, which was the least used codons of $E$. coli (Xu et al. 2006). To eliminate the influence of rare codons especially the least used codons, AGG, on the $\mathrm{Mu}$-IFN-CSP expression in recombinant $E$. coli, the $\mathrm{Mu}-\mathrm{IFN}-\mathrm{CSP}$ gene was codon-optimized according to the preferred codon usage of $E$. coli.

Many studies are devoted to produce functional interferon using DNA recombinant technology since IFNs have been applied as therapeutic medicine. Most of them have reported that the IFNs are expressed as inclusion bodies in recombinant E. coli (Neves et al. 2004; Srivastava et al. 2005; Valente et al. 2004). The refolding of misfolding and aggregation IFNs from inclusion bodies usually need extensive optimization, which makes the refolding procedures cumbersome (Middelberg 2002; Vu 

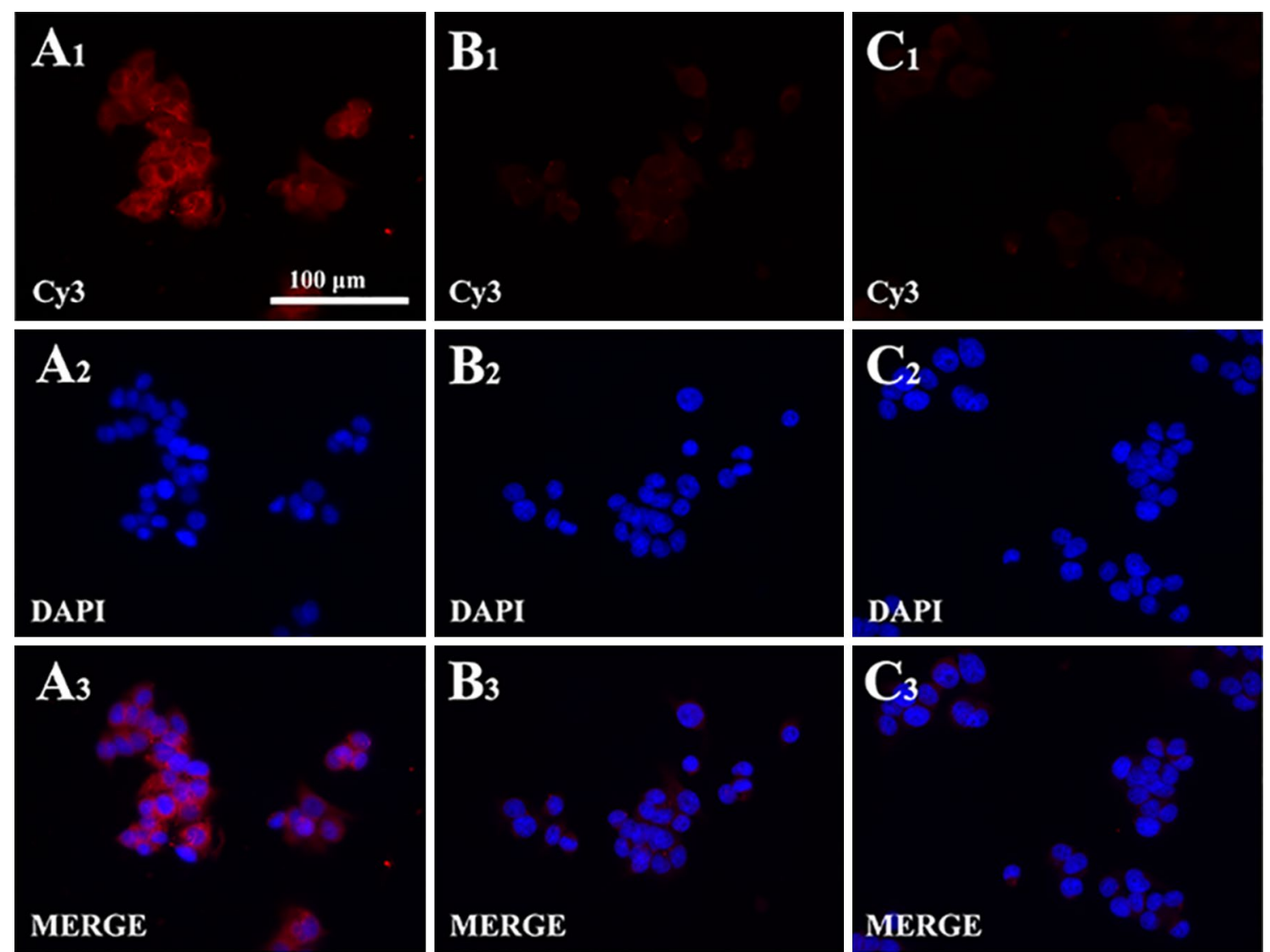

\section{DAPI}

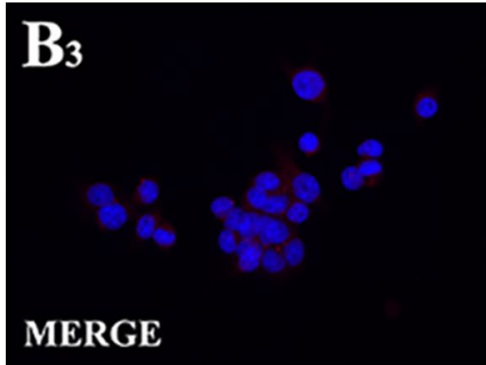

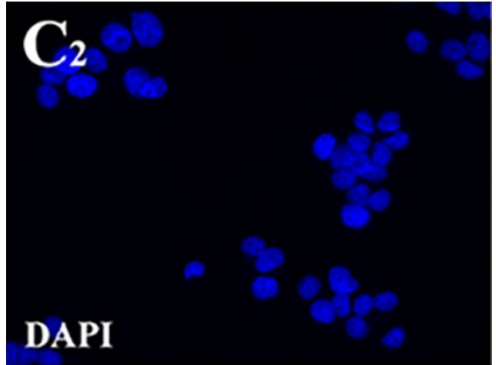

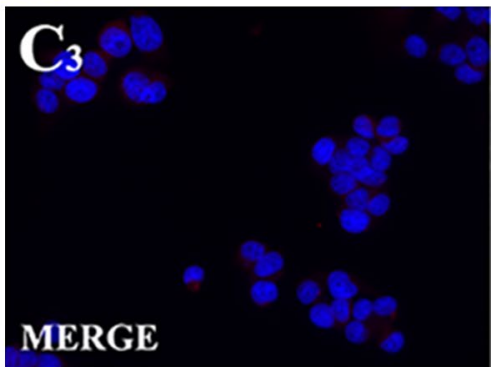

Fig. 9 Effect of IFN-CSP or mu-IFN-CSP on HBsAg expression in HepG2.2.15 cells. HepG2.2.15 cells stained by immunofluorescent staining with anti-HBsAg antibody and representative photographs are captured by microscope. A Controls; B IFN-CSP; C mu-IFN-CSP; 1 red stained with HBsAg; $\mathbf{2}$ blue nuclear stained with DAPI; $\mathbf{3}$ merged images of 1 and 2. Bar, $100 \mu \mathrm{m}$ and is the same for all photomicrographs

et al. 2016). Up to now, it is still hard to produce soluble IFNs in recombinant E. coli. In our previous study, the IFN-CSP is also expressed as inclusion bodies even the codon optimization strategy and expression conditions optimization strategy have been used ( $\mathrm{Lu}$ et al. 2015b). Our present study reported for the first time that liver-targeting interferon mu-IFN-CSP can be expressed as soluble form with $75.2 \%$ solubility. In further efforts to increase the soluble expression of mu-IFN-CSP in recombinant $E$. coli, the induction conditions for expression were optimized by an orthogonal test. As reported previously, optimization of induction conditions could be an effective strategy to improve the amount of various recombinant products in the soluble form (Liu et al. 2011; Yan et al. 2012). The present study showed that the solubility of mu-IFN-CSP was $75.2 \%$ without any induction conditions optimization. After induction conditions optimized, the solubility of mu-IFN-CSP was up to $98.4 \%$. The most optimal condition was at $\mathrm{OD}_{600}=0.9$, inducing the culture at $34{ }^{\circ} \mathrm{C}$ for $6 \mathrm{~h}$ with $0.1 \mathrm{mM}$ IPTG, which is consistent with previous reports that low temperature and low IPTG concentration may improve the solubility of target protein (Vu et al. 2016; Xu et al. 2006).

To study the influence of amino acid positions mutations on the function of mu-IFN-CSP, we also compared the liver tissue binding capacity and in vitro anti-HBV activity of the mutant with the native IFN-CSP. The results of in vitro liver tissue binding study shows that both the native protein and the mutant treatment displayed same degree green fluorescence, which indicate the mutation has no influence on the targeting capacity of the mu-IFN-CSP. The in vitro anti-HBV study in HepG2.2.15 cells showed that the soluble Mu-IFN-CSP has improved inhibition effect on HBV replication compared to the native IFN-CSP. These results are consistent with previous reports in which the mutation relates to the activity (Gangemi et al. 1989; Peek et al. 2004).

In summary, we designed a novel mu-IFN-CSP based on the native IFN-CSP using amino acid positions mutant. The structural comparison and molecular dynamic simulation showed that the $\mathrm{Mu}$ IFN-CSP formed three structure changes and were more 
stable than the native IFN-CSP. After amino acid mutant, codon-optimization and induction conditions optimization, the solubility of target protein was up to $98.4 \%$. Tissue sections binding and in vitro anti-HBV activity assays demonstrated that the soluble Mu-IFN-CSP has comparable liver tissue binding capacity and higher anti-HBV efficacy compared with the native IFN-CSP. The article reports for the first time that liver-targeting interferon $\mathrm{Mu}$-IFN-CSP can be expressed as soluble form, and also contributes to further support its application as liver-targeting anti-HBV medicine.

\begin{abstract}
Abbreviations
HBV: hepatitis B virus; E. coli: Escherichia coli; IFN: interferon; MD: molecular dynamic; RMSD: root mean square deviation; SOE-PCR: splicing by overlapping extension polymerase chain reaction; LB: Luria-Bertani; IPTG: isopropylthio-D-galactoside; SDS-PAGE: sodium dodecyl sulfate polyacrylamide gel electrophoresis; S: soluble supernatant; P: precipitation; LPS: lipopolysaccharides; RP-HPLC: reverse phase high-performance liquid chromatography; DAPI: 4',6-diamidino-2-phenylindole; HBsAg: hepatitis B s antigen; HBeAg: hepatitis $B$ e antigen; ELISA: enzyme-linked immunosorbent assay; FQ-PCR: fluorescent quantification PCR; SEM: standard error of the mean; ANOVA: Analysis of Variance; HSV: herpes simplex virus; BVDV: bovine viral diarrhea virus; VSV: vesicular stomatitis virus.
\end{abstract}

\section{Authors' contributions}

ALL and XML performed research and wrote the manuscript. SQG and LZ collected and analyzed data. SQG, XBJ and JYZ participated in the design of the study. ZXC, YNT, and JW participated in the in vitro antiviral experiments. MZX and WBL prepared the tissue sections. All authors read and approved the final manuscript.

\begin{abstract}
Author details
${ }^{1}$ School of Basic Courses, Guangdong Pharmaceutical University, 280 Wai Huan Dong Road, Guangzhou Higher Education Mega Center, Guangzhou 510006, People's Republic of China. ${ }^{2}$ Guangdong Provincial Key Laboratory of Pharmaceutical Bioactive Substances, 280 Wai Huan Dong Road, Guangzhou Higher Education Mega Center, Guangzhou 510006, People's Republic of China. ${ }^{3}$ Pharmaceutical College, Guangdong Pharmaceutical University, 280 Wai Huan Dong Road, Guangzhou Higher Education Mega Center, Guangzhou 510006, People's Republic of China. ${ }^{4}$ Intensive Care Unit, Shenzhen Second People's Hospital, The First Affiliated Hospital of Shenzhen University, Shenzhen 518031, People's Republic of China.
\end{abstract}

\section{Competing interests}

The authors declare that they have no competing interests.

\section{Availability of data and materials}

All datasets supporting the conclusions of the manuscript were included in the article.

\section{Ethical approval and consent to participate}

All experimental procedures were approved by Guangdong Pharmaceutical University Animal Care and Use Committee (Guangzhou, China), and carried out in compliance with the "Guide for the Care and Use of Laboratory Animals" published by the National Institutes of Health (US). This article does not contain any studies with human participants by any of the authors.

\section{Funding}

This work was financially supported by Science and Technology Planning Project of Guangzhou Municipality (No. 201607010272); Special Foundation of Public Welfare Research and Capacity Building of Guangdong Province (Nos. 2017A010105012; 2016A030303060); National Major Scientific and Technological Special Project for "Significant New Drugs Development" of China (2013ZX09103003-003); National Natural Science Foundation of China (No. 31501894); Natural Science Foundation of Guangdong Province of China
(No. 2015A030310036); Medical Scientific Research Foundation of Guangdong Province of China (No. A2016091); the Joint Natural Sciences Fund of the Department of Science and Technology and the First Affiliated Hospital of Guangdong Pharmaceutical University (No. GYFYLH201312).

\section{Publisher's Note}

Springer Nature remains neutral with regard to jurisdictional claims in published maps and institutional affiliations.

Received: 11 September 2017 Accepted: 25 October 2017

Published online: 30 October 2017

\section{References}

Bao D, Jin X, Ma Y, Zhu J (2015) Comparison of the structure and biological activities of wild-type and mutant liver-targeting peptide modified recombinant human endostatin (rES-CSP) in human hepatocellular carcinoma HepG2 Cells. Protein Pept Lett 22(5):470-479

Degasperi E, Aghemo A, Colombo M (2017) Treatment of extrahepatic manifestations of hepatitis C virus. Clin Liver Dis 21(3):631-643. https://doi. org/10.1016/..cld.2017.03.015

Gangemi JD, Lazdins J, Dietrich FM, Matter A, Poncioni B, Hochkeppel HK (1989) Antiviral activity of a novel recombinant human interferon-alpha B/D hybrid. J Interferon Res 9(2):227-237

Ghaffar A, Mayer EP, Barnhart DC, Pyo S, Szymaniec S, Hochkeppel HK, Gangemi JD (1992) Cross-species antiviral activity of a recombinant human alpha-interferon hybrid. Ann NY Acad Sci 653:314-322

Goeddel DV, Yelverton E, Ullrich A, Heyneker HL, Miozzari G, Holmes W, Seeburg PH, Dull T, May L, Stebbing N, Crea R, Maeda S, McCandliss R, Sloma A, Tabor JM, Gross M, Familletti PC, Pestka S (1980) Human leukocyte interferon produced by $E$. coli is biologically active. Nature 287(5781):411-416

Gull I, Samra ZQ, Aslam MS, Athar MA (2013) Heterologous expression, immunochemical and computational analysis of recombinant human interferon alpha 2b. Springerplus 2(1):264. https://doi. org/10.1186/2193-1801-2-264

Horisberger MA, de Staritzky K (1987) A recombinant human interferon-alpha B/D hybrid with a broad host-range. J Gen Virol 68(Pt 3):945-978. https:// doi.org/10.1099/0022-1317-68-3-945

Karpusas M, Nolte M, Benton CB, Meier W, Lipscomb WN, Goelz S (1997) The crystal structure of human interferon beta at 2.2-A resolution. Proc Natl Acad Sci USA 94(22):11813-11818

Kato S (2015) Molecular targeted drugs for soft tissue sarcoma and neuroendocrine tumor. Nihon Rinsho 73(8):1398-1402

Kotredes KP, Thomas B, Gamero AM (2017) The protective role of type I interferons in the gastrointestinal tract. Front Immunol 8:410. https://doi. org/10.3389/fimmu.2017.00410

Liu JF, Zhang ZJ, Li AT, Pan J, Xu JH (2011) Significantly enhanced production of recombinant nitrilase by optimization of culture conditions and glycerol feeding. Appl Microbiol Biotechnol 89(3):665-672

Lu XM, Jin XB, Zhu JY, Mei HF, Ma Y, Chu FJ, Wang Y, Li XB (2010) Expression of the antimicrobial peptide cecropin fused with human lysozyme in Escherichia coli. Appl Microbiol Biotechnol 87(6):2169-2176

Lu XM, Jin XB, Huang YT, Wang J, Shen J, Chu FJ, Mei HF, Ma Y, Zhu JY (2014) Construction of a novel liver-targeting fusion interferon by incorporation of a Plasmodium region I-plus peptide. Biomed Res 2014:e261431

Lu X, Wang J, Jin X, Huang Y, Zeng W, Zhu J (2015a) IFN-CSP inhibiting hepatitis B virus in HepG2.2.15 cells involves JAK-STAT signal pathway. Biomed Res Int 2015:959684. https://doi.org/10.1155/2015/959684

Lu X, Wang J, Jin X, Zhu J (2015b) High-level expression of a novel liver-targeting fusion interferon with preferred Escherichia coli codon preference and its anti-hepatitis B virus activity in vivo. BMC Biotechnol 15:54. https://doi. org/10.1186/s12896-015-0177-1

Lu X, Wang J, Jin X, Huang Y, Zeng W, Zhu J (2016) Targeting mechanism of a novel liver-targeting interferon IFN-CSP involves liver heparan sulfate proteoglycan. Curr Drug Deliv 13(4):528-533

Meister A, Uze G, Mogensen KE, Gresser I, Tovey MG, Grutter M, Meyer F (1986) Biological activities and receptor binding of two human recombinant 
interferons and their hybrids. J Gen Virol 67(Pt 8):1633-1643. https://doi. org/10.1099/0022-1317-67-8-1633

Middelberg AP (2002) Preparative protein refolding. Trends Biotechnol 20(10):437-443

Neves FO, Ho PL, Raw I, Pereira CA, Moreira C, Nascimento AL (2004) Overexpression of a synthetic gene encoding human alpha interferon in Escherichia coli. Protein Expr Purif 35(2):353-359

Peek SF, Bonds MD, Gangemi DG, Thomas CB, Schultz RD (2004) Evaluation of cytotoxicity and antiviral activity of recombinant human interferon alfa$2 \mathrm{a}$ and recombinant human interferon alfa-B/D hybrid against bovine viral diarrhea virus, infectious bovine rhinotracheitis virus, and vesicular stomatitis virus in vitro. Am J Vet Res 65(6):871-874

Peng L, Xu Z, Fang X, Wang F, Yang S, Cen P (2004) Preferential codons enhancing the expression level of human beta-defensin-2 in recombinant Escherichia coli. Protein Pept Lett 11(4):339-344

Radhakrishnan R, Walter LJ, Hruza A, Reichert P, Trotta PP, Nagabhushan TL, Walter MR (1996) Zinc mediated dimer of human interferon-alpha $2 b$ revealed by X-ray crystallography. Structure 4(12):1453-1463

Sharp PM, Li WH (1987) The codon adaptation index-a measure of directional synonymous codon usage bias, and its potential applications. Nucleic Acids Res 15(1987):1281-1295

Srivastava P, Bhattacharaya P, Pandey G, Mukherjee KJ (2005) Overexpression and purification of recombinant human interferon alpha2b in Escherichia coli. Protein Expr Purif 41(2):313-322
Suginoshita Y, Tabata Y, Moriyasu F, Ikada Y, Chiba T (2001) Liver targeting of interferon-beta with a liver-affinity polysaccharide based on metal coordination in mice. J Pharmacol Exp Ther 298(2):805-811

Valente CA, Prazeres DM, Cabral JM, Monteiro GA (2004) Translational features of human alpha 2b interferon production in Escherichia coli. Appl Environ Microbiol 70(8):5033-5036

Vu TT, Jeong B, Krupa M, Kwon U, Song JA, Do BH, Nguyen MT, Seo T, Nguyen AN, Joo CH, Choe H (2016) Soluble prokaryotic expression and purification of human interferon alpha-2b using a maltose-binding protein tag. J Mol Microbiol Biotechnol 26(6):359-368. https://doi. org/10.1159/000446962

Wang J, Lu X, Jin X, Zeng W, Zhu J (2015) Comparative pharmacokinetics, tissue distribution, excretion of recombinant liver-targeting interferon with IFN alpha2b administered intramuscular in rats. Curr Pharm Biotechnol 16(10):911-919

Xu Z, Zhong Z, Huang L, Peng L, Wang F, Cen P (2006) High-level production of bioactive human beta-defensin-4 in Escherichia coli by soluble fusion expression. Appl Microbiol Biotechnol 72(3):471-479

Yan X, Hu S, Guan YX, Yao SJ (2012) Coexpression of chaperonin GroEL/GroES markedly enhanced soluble and functional expression of recombinant human interferon-gamma in Escherichia coli. Appl Microbiol Biotechnol 93(3):1065-1074

\section{Submit your manuscript to a SpringerOpen ${ }^{\odot}$ journal and benefit from:}

- Convenient online submission

- Rigorous peer review

- Open access: articles freely available online

- High visibility within the field

- Retaining the copyright to your article

Submit your next manuscript at $\boldsymbol{\nabla}$ springeropen.com 